Lyle, M., Wilson, P.A., Janecek, T.R., et al., 2002

Proceedings of the Ocean Drilling Program, Initial Reports Volume 199

\title{
7. Geochemical Analysis of Bulk MARINE SEDIMENT BY INDUCTIVELY Coupled Plasma-Atomic Emission SPECTROSCOPY ON BOARD THE JOIDES RESOLUTION ${ }^{1}$
}

\author{
L.L. Quintin, ${ }^{2}$ Kristina L. Faul, ${ }^{3}$ Caroline Lear, ${ }^{4}$ Dennis Graham, ${ }^{5}$ \\ Chieh Peng, ${ }^{5}$ R.W. Murray, ${ }^{6}$ and Shipboard Scientific Party ${ }^{5}$
}

\begin{abstract}
Geochemical analyses on board the JOIDES Resolution have been enhanced with the addition of a Jobin-Yvon Ultrace inductively coupled plasma-atomic emission spectrometer (ICP-AES) as an upgrade from the previous X-ray fluorescence facility. During Leg 199, we sought to both challenge and utilize the capabilities of the ICP-AES in order to provide an extensive bulk-sediment geochemical database during the cruise. These near real-time analyses were then used to help characterize the recovered sedimentary sequences, calculate mass accumulation rates of the different sedimentary components, and assist with cruise and postcruise sampling requests. The general procedures, sample preparation techniques, and basic protocol for ICP-AES analyses on board ship are outlined by Murray et al. (2000) in Ocean Drilling Program Tech Note, 29. We expand on those concepts and offer suggestions for ICPAES methodology, calibration by standard reference materials, data reduction procedures, and challenges that are specific to the analysis of bulk-sediment samples. During Leg 199, we employed an extensive bulk-sediment analytical program of $\sim 600$ samples of varying lithologies, thereby providing several opportunities for refinement of techniques. We also discuss some difficulties and challenges that were faced
\end{abstract}

${ }^{1}$ Examples of how to reference the whole or part of this volume.

${ }^{2}$ Department of Earth Sciences, Boston University, Boston MA 02215, USA. quintin@bu.edu

${ }^{3}$ Department of Geological \&

Environmental Sciences, Stanford University, Stanford CA 94305-2115, USA.

${ }^{4}$ Institute of Marine and Coastal Sciences, Rutgers University, New Brunswick NJ 08901, USA.

${ }^{5}$ Shipboard Scientific Party addresses.

${ }^{6}$ Department of Earth Sciences, Boston University, Boston MA 02215, USA. 
L.L. QUINTIN ET AL.

and suggest how to alleviate such occurrences for sedimentary chemical analyses during future legs.

\section{INTRODUCTION}

An important component of the seagoing studies of Ocean Drilling Program (ODP) Leg 199 involved the geochemical analysis of bulk sediments in order to provide chemical analyses while at sea. Our sampling plan was ambitious and resulted in $~ 600$ samples being analyzed during the cruise for a suite of 10 elements $(\mathrm{Si}, \mathrm{Al}, \mathrm{Ti}, \mathrm{Fe}, \mathrm{Mn}, \mathrm{Ca}, \mathrm{Mg}, \mathrm{P}, \mathrm{Ba}$, and $\mathrm{Sr}$ ). These analyses allowed for first-order paleoceanographic decisions (e.g., sampling the Paleocene/Eocene $[\mathrm{P} / \mathrm{E}]$ boundary) to be made and drilling objectives to be optimized, while also allowing shipboard scientists to tailor their cruise and postcruise sampling requests and shorebased research. Whereas chemical analysis of the bulk sediment provides reinforcement for the large-scale lithologic changes, it can also further provide information on small-scale lithologic changes that may otherwise go unnoticed.

For many years, the analysis of major and trace elements on board the JOIDES Resolution was performed using X-ray fluorescence (XRF) spectrometry and focused primarily on igneous rocks. This instrument has been subsequently replaced by a Jobin Yvon 2000 sequential inductively coupled plasma-atomic emission spectrometer (ICP-AES) (Murray et al., 2000). General ICP-AES procedures, analyses information, and preparation procedures are described in Murray et al. (2000). The shipboard ICP-AES was first used for igneous rock (during Leg 187) and interstitial water (during Legs 188 and 189) analyses. In particular during Leg 187 (Australian-Antarctic Discordance), real-time drilling decisions were made using the ICP-AES to help select, while at sea, specific sites to drill (Christie Pederson, Miller, et al., 2001).

Despite these successes with ICP-AES analyses of igneous rocks and pore waters, until Leg 199 the ideal opportunity to use the instrument for quantitative analysis of sediment had not been realized. Shipboard major and trace element analyses provide an initial chemical characterization of the bulk sediment that can then be further expanded during shore-based analyses. For Leg 199, this chemical characterization further fuels the initial development and interpretation of mass accumulation rates (MARs), Intertropical Convergence Zone migration, and paleoceanographic changes occurring in the equatorial Pacific throughout the Paleogene. These ideas and concepts can then be revised and reinterpreted during shore-based treatment without the need to wait for basic chemical analyses. Whereas a more complete major, trace, and rare earth element analysis of the bulk sediment and eolian fraction can wait for shore-based study, analyses on board the JOIDES Resolution provide a first-order analysis useful to the entire shipboard and shore-based scientific party.

Leg 199 was particularly appropriate to use as a test bed for sedimentary ICP-AES analysis because of the variety of lithologies recovered and analytical opportunities, including analysis of the P/E boundary. Red clay, radiolarian clay, radiolarite, nannofossil ooze, nannofossil chalk, dolomite, and other sediment types were encountered. Each exhibited varying geochemical signatures. Small changes in Si or Ca concentration, for example, are easily tracked by the geochemical analyses but when viewed from the sedimentological perspective often go unno- 
L.L. QUINTIN ET AL.

ticed. Analysis by ICP-AES is a complement to the sedimentological interpretations but also provides clues that can be easily missed.

\section{ANALYTICAL METHODOLOGY}

\section{Elemental Menu}

$\mathrm{Si}, \mathrm{Al}, \mathrm{Ti}, \mathrm{Fe}, \mathrm{Mn}, \mathrm{Ca}, \mathrm{Mg}, \mathrm{P}, \mathrm{Ba}$, and Sr were analyzed. Although this element menu is less extensive than the common igneous suite (Murray et al., 2000), this specific suite was constructed to provide critical information on terrigenous abundance (based on $\mathrm{Al}$ and $\mathrm{Ti}$ ), the oxide metalliferous component ( $\mathrm{Fe}$ and $\mathrm{Mn}$ ), and biogenic input ( $\mathrm{Si}, \mathrm{Ca}, \mathrm{P}, \mathrm{Ba}$, and $\mathrm{Sr}$ ). Although additional elements could have been analyzed (e.g., $\mathrm{Zr}$ ), we limited the protocol to 10 elements to maximize sample throughput. The measured wavelengths for the targeted elements are recorded in Table $\mathbf{T} 1$.

\section{Sample Frequency And Preparation}

Bulk-sediment samples were taken at a frequency of one $2.5-\mathrm{cm}^{3}$ sample per section of core (approximately every $1.5 \mathrm{~m}$ ) for Sites 12151217. To conserve shipboard argon, used in record quantity during the leg, samples were taken at a frequency of three samples per core (Sections 2, 4, and 6) for Sites 1218-1222. All samples were taken adjacent to physical properties samples to provide the most complete data set for the calculation of chemical accumulation rates that are based in part upon dry bulk density values measured on the physical properties samples.

Bulk samples were prepared according to the method of Murray et al. (2000). Although the procedure was originally developed and optimized for analysis of igneous rocks, we found that the sample masses used $(0.1 \mathrm{~g})$, the flux mass $(0.4 \mathrm{~g})$, and other aspects of the preparation protocol worked well for the diverse sedimentary lithologies recovered during Leg 199 (see "Calibration Standards," p. 4). It will be apparent from the discussions below of in-run, in-site, and long-term precision that procedural differences were not a significant factor in the outcome of the analyses. Refer to Murray et al. (2000) and the "Explanatory Notes" chapter for preparation and procedure.

\section{Selection of Solution to Monitor Instrumental Drift}

Compared with ICP-AES analyses of igneous rocks and interstitial waters, sediment analyses require a strict control on analytical drift because of the variability and low concentrations of specific elements in the sediment. A drift solution must be analyzed multiple times throughout an analytical run in order to account and correct for instrumental drift during data reduction. Typical drift should be between $\pm 1 \%$ and $\pm 5 \%$, which is $\sim 1 \%$ per hour of analyses. The drift solution must be matrix matched for the most accurate and precise results (Murray et al., 2000). This presented a particular challenge for the sedimentary analyses during this leg given the variety of lithologies encountered. We found, however, that overall drift for each element (except P, which is discussed later) was $<5 \%$. We used the igneous rock standard K1919 (Kilauea basalt) for drift corrections. Although not a sedimentary rock, we found no matrix-induced effects in using this material. Using $\overline{\text { T1. Analytical information, p. } 14 \text {. }}$ 
L.L. QUINTIN ET AL.

this drift solution also helped to assess long-term (interleg) behavior of the ICP-AES instrument, as this is the same drift solution used in igneous rock protocols.

\section{Mass Corrections}

During data reduction, one must account for instrumental drift as measured by the drift solution and background counts as measured by the analytical blank solution as well as differences in sample weight for each analysis. In order to account for differences in sample and acid weight during data reduction, flux and sample must be weighed to a precision of $0.5 \%$ of the measured value, which can be accomplished by weighing within a range of 0.3995-0.4005 $\mathrm{g}$ for the flux and 0.0995$0.1005 \mathrm{~g}$ for the sample. To correct for the weight differences during data reduction, the values are recorded and the weight factor is determined based on a value of one (1.00) being idealized $4000 \times$ dilution as indicated by Murray et al. (2000). This value is then multiplied by the drift-corrected counts before being used in the calibration regression.

\section{Calibration Standards}

ICP-AES is a comparative analytical technique. In order to convert the counts as provided by the instrument into concentrations, standard reference materials (SRMs) are prepared in the same manner as the samples and analyzed within each separate run. For Leg 199, SRMs were prepared once at the beginning of the leg in one batch following the procedure outlined above and in Murray et al. (2000). The integrity and stability of the solutions remained strong throughout the cruise, as indicated by the consistent and highly linear calibrations. The drift- and blank-corrected counts were then regressed against the known concentrations for each of the elements for the standards (Fig. F1). SRMs were run as separate samples in each analytical run. This calibration must be done for each element for each analytical run. Although we used an SRM for the drift correction (K1919), we did not use this SRM in the calibration.

The four or five SRMs selected must cover the anticipated high and low concentrations for all of the elements that will be analyzed. Again, the diversity of recovered lithologies of Leg 199 provided a particular challenge, both in terms of covering the range of elemental concentrations and being aware of possible matrix affects. We chose Cody shale (SCo-1), Pahoehoe basalt lava flow (BHVO-2), and marine mud (MAG1) from the U.S. Geological Survey and argillaceous limestone (NIST-1C) from the U.S. National Institute of Standards and Technology. This particular suite was selected in order to accommodate the chert, radiolarite, carbonate, and clay-rich sediments. We modified the procedures of Murray et al. (2000) to optimize the results specifically for bulk sediments. The concentration of the analytical blanks is considered zero and was used in the calibration regression. An alternative would be to perform a blank subtraction and force the regression through the origin $(0,0)$; however, we found that using the blank as a default zero yielded the best results. Detection limits for each of the elements analyzed are also based on the blank analyses (Table T1).

The strong linearity (e.g., 0.999-1.000) of the calibrations (Fig. F1) indicates that the above suite of chosen SRMs provides an excellent calibration protocol appropriate for the majority of the range of sediment lithologies. This suite of SRMs is likely to be appropriate for other legs,
F1. Calibrations for $\mathrm{Al}$ and Fe, p. 10.
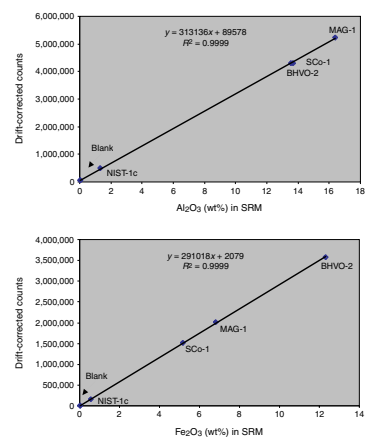
L.L. QUINTIN ET AL.

as well. However, for legs that may encounter carbonate-rich lithologies (see "Difficulties and Challenges," p. 5), it is recommended that a pure $\mathrm{CaCO}_{3}$ powder be added to the SRM menu.

\section{Precision}

Precision is measured both short term within a single analytical run, between several runs at one site, and long term from site to site throughout the leg (Table T1). The ICP-AES analyzes each element in a given solution between one and five (this is a parameter set by the analyst) and is most commonly selected to analyze in triplicate. This provides a measurement of precision for each element for each analyzed sample, and is reported as a percent relative standard deviation (\%RSD). The \%RSD improves over time within a run as the instrument continues to warm up and equilibrate with its surroundings. Typical shipboard \%RSD is between $1 \%$ and $2 \%$.

Within-run precision is measured by repeated, or replicate, analyses of one particular solution. For the shipboard Leg 199 analysis, the SRM Japanese chert (JCh-1) was used as the replicate solution in order to conserve precious sample, argon, and preparation time (see "Difficulties and Challenges," p. 5, regarding argon usage). Typical precision values for individual analyses were between 1\% and 8\% (except for $\mathrm{P}$, as discussed below). For shore-based analytical runs, it is standard practice to prepare two or more identical samples using the same procedure and then analyze them all in one run, which quantifies both precision of the instrument and of the preparation procedure. Because of logistical concerns (e.g., consumption of Ar), we did not use that strategy. We also used the drift solution to calculate the precision between several analytical runs performed at a single site, in addition to the individual run precision. This helps to further constrain the data and detect problems. Drift solution (K1919) was not included in the calibration and was treated as a sample in the run, which allowed us to use its values to determine site precision. Typical site precision values for Leg 199 were between $0.7 \%$ and $4 \%$ (except for $\mathrm{P}$, as discussed below). Long-term precision is determined by calculating the average and standard deviation (on a per element basis) of all the data on this solution (made from an identical powder) throughout the leg.

As the Al analyses from each site show (Fig. F2), precision does not necessarily improve with increased number of analytical runs. The overall (site) precision of analyses for all elements, except $\mathrm{P}$, is within $0.5 \%-15 \%$ of the measured value, which is quite acceptable given the difficult sample preparation and analytical environment at sea (compared to shore-based precision, which is typically $0.5 \%-2 \%$ ). Phosphorous has greater variability in its precision (between $8 \%$ and $67 \%$ of the measured value) as a result of nitrogen flow; this is discussed in greater detail later in this paper (see "Difficulties and Challenges," p. 5). Overall, long-term precision ranges between $3 \%$ and $21 \%$ for all elements excluding $\mathrm{P}$, which is $\sim 33 \%$.

\section{DIFFICULTIES AND CHALLENGES}

As with any analytical protocol, a cavalcade of unanticipated difficulties associated with seagoing chemical analyses often arises to cause a change in the guidelines and data-reduction techniques of an analytical run. Shipboard analyses provide a different situation than shore-based
F2. Precision of $\mathrm{Al}$ analyses, p. 11.

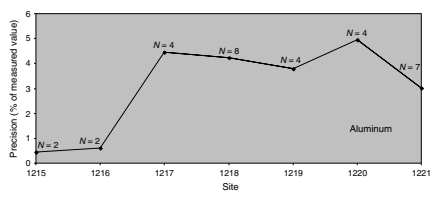


L.L. QUINTIN ET AL.

analyses, where increased error can be introduced because of powder weighing, time constraints, increased variability in lithologies, and availability of materials.

Weighing of sample and lithium metaborate flux on board the JOIDES Resolution is relatively accurate when seas are calm. However, when seas are rough, it is difficult to accurately weigh without significant error. Flux, for the most part, was weighed previously on shore at Texas A\&M University to save time; these values are relatively accurate. Flux-powder weight is not as critical as sample powder weight; therefore, the greatest error in weighing is introduced by inaccuracy in sample weight. Even though a weight correction is applied to the data, there is still an error that can not be accounted for. Although igneous geochemists tend to analyze relatively fewer samples (albeit, for a more comprehensive element menu), the analysis of hundreds of sediments on a given leg strictly requires that the flux aliquots be preweighed on shore.

ICP-AES analyses during transit and high seas cause problems that concern high drift, low precision and accuracy, and increased \%RSD values. We observed an obvious contrast between instrumental operation during transit and instrumental operation while at site, with significantly poorer results occurring during the transit. We believe this poorer precision is due to the mechanism of nebulization. The nebulizer generates an aerosol mist and injects humidified Ar gas into the nebulizer along with the sample. The ICP-AES on board the JOIDES Resolution is bolted to a table to avoid movement of the instrument, and this is directly coupled to the ship's movement. Upon injection into the spray chamber, however, the aerosol is decoupled from the instrument, which moves around the aerosol (from the aerosol's reference frame). This creates a situation where the aerosol is inconsistently sampled by the plasma, resulting in high drift and low precision for a run. For example, data from Site 1222, which was analyzed during a transit, experienced drift between samples of $50 \%$ or more, and the standard deviation for each element concentration and sample was so large that no true pattern was apparent.

The precision of the analyses of $\mathrm{P}$ indicated low reproducibility between analytical runs (Table T1). Phosphorus requires the input of nitrogen gas to improve detection limits during sampling because of its low-emission wavelength, which is severely compromised by interference with air. A relatively new nitrogen system on board the JOIDES Resolution was employed for Sites 1215-1219, where nitrogen was supplied to the instrument directly from a nitrogen generator rather than from the storage cylinders. Initially, it was thought that the flow of nitrogen from the generator would be the best source because there would be no period of decreased nitrogen as there would be if a cylinder began to deplete. However, after various tests, we determined that nitrogen supplied via the generator caused precision to greatly decrease $(\sim 25 \%)$, and nitrogen supplied via the storage cylinders (Sites 1220$1221)$ caused precision to improve $(\sim 10 \%)$. Again, this nitrogen flush is only relevant for $\mathrm{P}$ because other elements are unaffected by the atmospheric interference.

During the leg, we encountered $\mathrm{CaCO}_{3}$-rich sediments (nannofossil ooze/chalk) that, when analyzed, indicated $\mathrm{Ca}$ values $>40 \%$, which would indicate $>100 \% \mathrm{CaCO}_{3}$. This is obviously impossible and is probably due to the fact that Ca concentrations in the SRMs used in the calibration did not span the concentration range of the samples measured. Our highest SRM for Ca values was NIST-1C with just over $35 \% \mathrm{Ca}$, 
L.L. QUINTIN ET AL.

which is significantly less than a pure $\mathrm{CaCO}_{3}$ end-member. Also, weighing errors may have also played a role. Regardless, for future legs targeting carbonate-rich sediments, we suggest the use of a pure calcium carbonate powder as a high-end calibration for $\mathrm{Ca}$. Since this was seen in only a few samples $(\sim 5-10)$, it did not prove to be a significant thorn in the side of the shipboard program, and further shore-based analyses will help to constrain the problem.

Sample throughput was limited by the supply of Ar gas and the number of platinum crucibles. We recommend that legs considering a large ICP-AES analytical program should include sufficient Ar gas tanks as well as additional platinum crucibles. This will not only increase sample throughput but will also allow for increased analysis of standards, replicates for determination of precision, and other parameters that will improve the overall results.

\section{REPRESENTATIVE RESULTS}

Individual results of the geochemical analysis of the bulk sediment for each site studied during Leg 199 are recorded in the site chapters in this volume and, therefore, are not discussed in detail here. However, we illustrate the utility of bulk-sediment analysis by ICP-AES on board the JOIDES Resolution by presenting representative profiles of $\mathrm{Si}$ and $\mathrm{Ca}$ (weight percent of sediment) from Site 1218 as compared to observed lithologic units (Fig. F3). The concentration of silica increases in radiolarite and is relatively low in the nannofossil ooze and chalk. The geochemical analysis of the bulk sediment supports the identified lithologic units, and it also provides the concentrations of silica and calcium (for example) that become necessary when distinguishing nannofossil ooze and chalk. Small-scale variations in composition within each unit are also apparent.

When used in conjunction with the concentration of Ti or another terrigenous-associated metal, bulk-sediment geochemical analyses of Si and $\mathrm{Ca}$ can be used as a basis for normative calculations of the concentration of biogenic opal (biogenic $\left.\mathrm{SiO}_{2}\right)$ and calcium carbonate $\left(\mathrm{CaCO}_{3}\right)$, as well as for initial estimations of biogenic and terrigenous MARs. Coupled with other shipboard analyses and a preliminary age model, each element characterized provides a first-order interpretation of influences on export production, climate, and terrigenous input (via Ti) in the equatorial Pacific (Fig. F4). This then provides a basis for more extensive, specific, and detailed shore-based research by the shipboard and shore-based parties.

\section{SUMMARY}

In this brief contribution, we have provided guidelines that are specific to sediment analysis on board the JOIDES Resolution. The geochemical bulk-sediment data set that accompanied the shipboard party upon the completion of Leg 199 highlights the opportunity this facility presents to the sedimentary and paleoceanographic community. We have shown that it is possible to analyze hundreds of samples with an acceptable degree of precision, and we have demonstrated the utility of the data for first-order paleoceanographic interpretations. Preliminary results can be provided in hours instead of days by XRF or months by shore-based analyses. We hope that continued use of the ICP-AES for
F3. Silicon and $\mathrm{Ca}$ as a function of depth, p. 12.

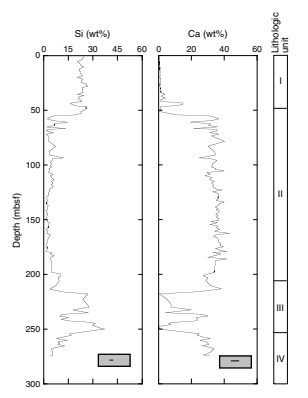

F4. Silicon, $\mathrm{Ca}$, and Ti and MAR vs. age and lithology, p. 13.

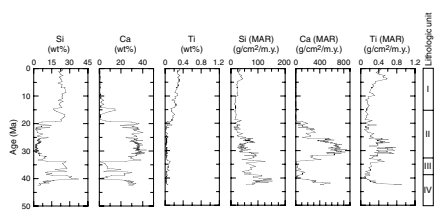


L.L. QUINTIN ET AL.

Chapter 7, Geochemical Analysis of Bulk Marine Sediment

8

bulk-sediment analyses while on board the JOIDES Resolution will help to expand the goals and prospects of upcoming cruises.

ACKNOWLEDGMENTS

This research used samples and/or data provided by the Ocean Drilling Program (ODP). ODP is sponsored by the U.S. National Science Foundation (NSF) and participating countries under management of Joint Oceanographic Institutions (JOI), Inc. Funding for this research was provided by the NSF (EAR97-24282, OCE98-18713) for acquisition of the shore-based ICP-AES facilities at Boston University, where these protocols were initially developed for ODP.

We thank the Co-Chief Scientists of Leg 199, Mitch Lyle and Paul Wilson, for their strong support and interest in bulk-sediment analyses by ICP-AES. Their enthusiasm for this project was vital to its completion. RWM acknowledges GRD for The Words. We thank Peggy Delaney and Mitch Lyle for very many helpful comments on the manuscript. 


\section{L.L. QUINTIN ET AL.}

Chapter 7, Geochemical Analysis of Bulk Marine Sediment

\section{REFERENCES}

Christie, D.M., Pedersen, R.B., Miller, D.J., et al., 2001. Proc. ODP, Init. Repts., 187 [CDROM]. Available from: Ocean Drilling Program, Texas A\&M University, College Station TX 77845-9547, USA.

Murray, R.W., Miller, D.J., and Kryc, K.A., 2000. Analysis of major and trace elements in rocks, sediments, and interstitial waters by inductively coupled plasma-atomic emission spectrometry (ICP-AES). ODP Tech. Note, 29 [Online]. Available from World Wide Web: <http://www-odp.tamu.edu/publications/tnotes/tn29/ INDEX.HTM>. [Cited 2002-07-23] 


\section{L.L. QUINTIN ET AL.}

Chapter 7, Geochemical Analysis of Bulk Marine Sediment

Figure F1. Representative calibrations for $\mathrm{Al}$ and Fe from Site 1218. The four standard reference materials used in the calibration (BHVO-2 = Pahoehoe basalt lava flow, MAG-1 = marine mud, SCo-1 = Cody shale, NIST-1c $=$ argillaceous limestone) are shown along with the blank analyses.
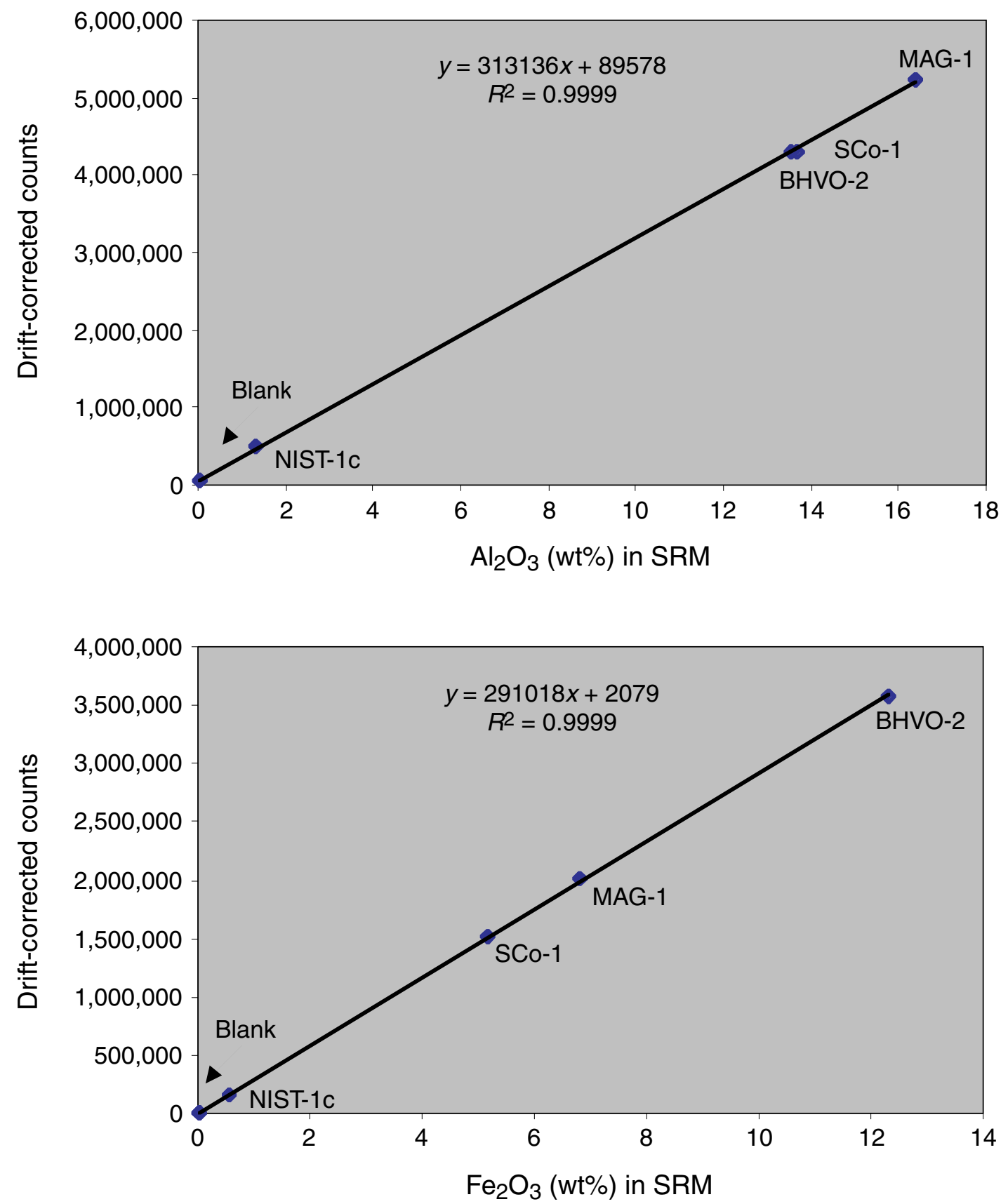


\section{L.L. QUINTIN ET AL.}

Chapter 7, Geochemical Analysis of Bulk Marine Sediment

Figure F2. Precision of Al analyses over the duration of Leg 199, beginning with Site 1215 and ending with Site 1221. ICP-AES samples for Site 1222 were not accounted for because of reasons stated within the text (see "Difficulties and Challenges," p. 5). $N=$ number of analytical runs done at that particular site from which precision was determined.

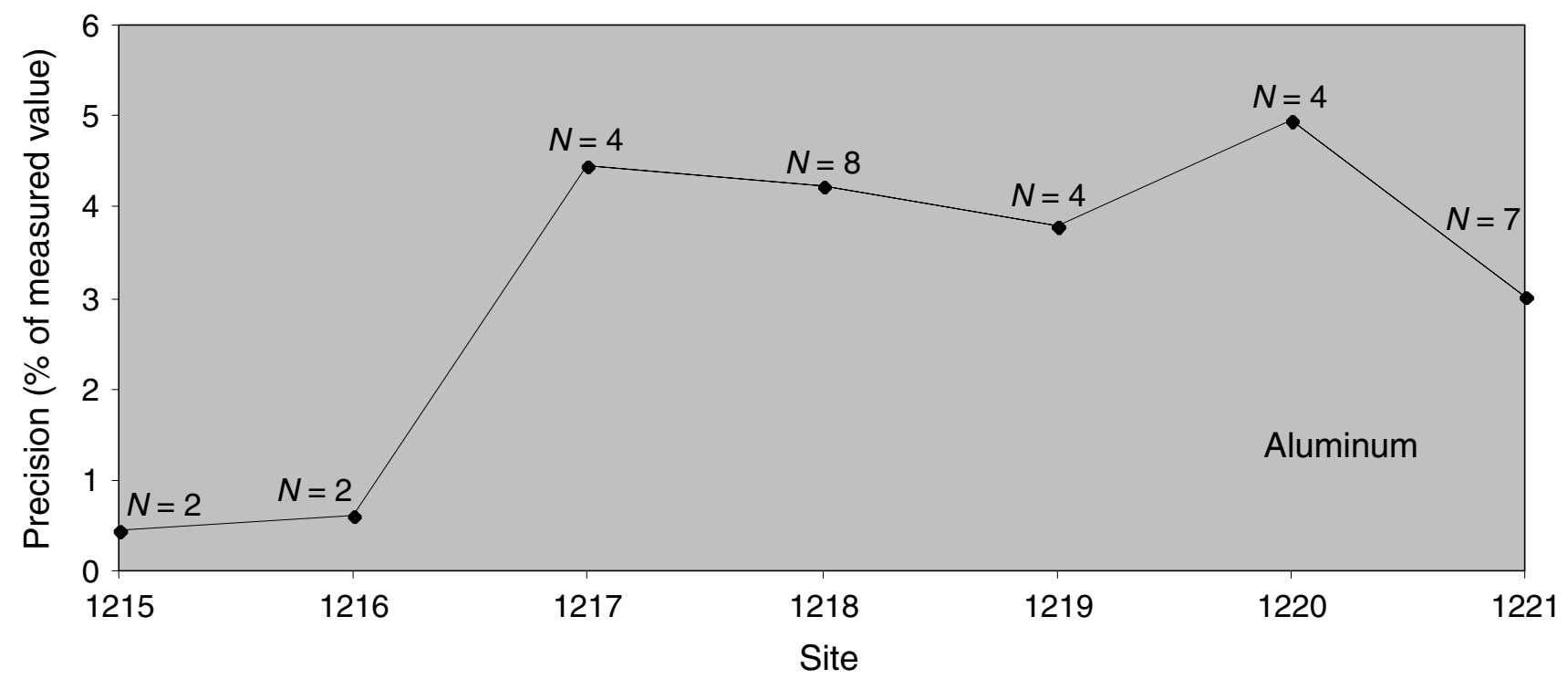




\section{L.L. QUINTIN ET AL.}

Figure F3. Si and Ca expressed as weight percent of the sediment from Site 1218 as a function of depth. The relationship to lithologic units (I = clay, II = nannofossil ooze/chalk, III = radiolarite, IV = nannochalk/ dolomite) is also shown. Each point indicates a separate chemical analysis. The line inside the gray box in the bottom right corner of each graph indicates the long-term precision of the analysis (see Table T1, p. 14).

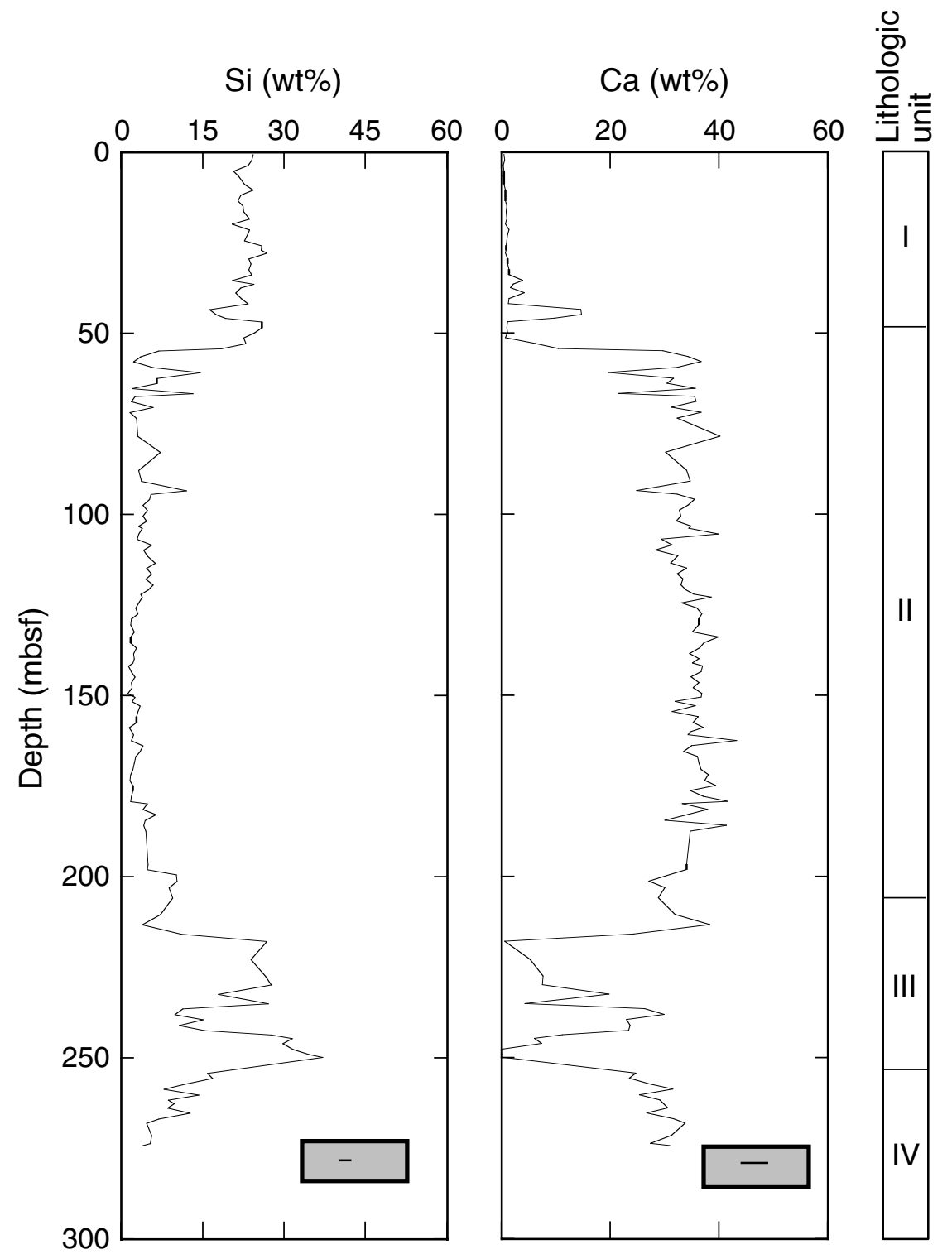




\section{L.L. QUINTIN ET AL.}

Chapter 7, Geochemical Analysis of Bulk Marine Sediment

Figure F4. Si, Ca, and Ti expressed as weight percent of the sediment and as a mass accumulation rate (MAR) vs. age and lithology for Site 1218. Lithologies are those stated in Figure F3, p. 12.

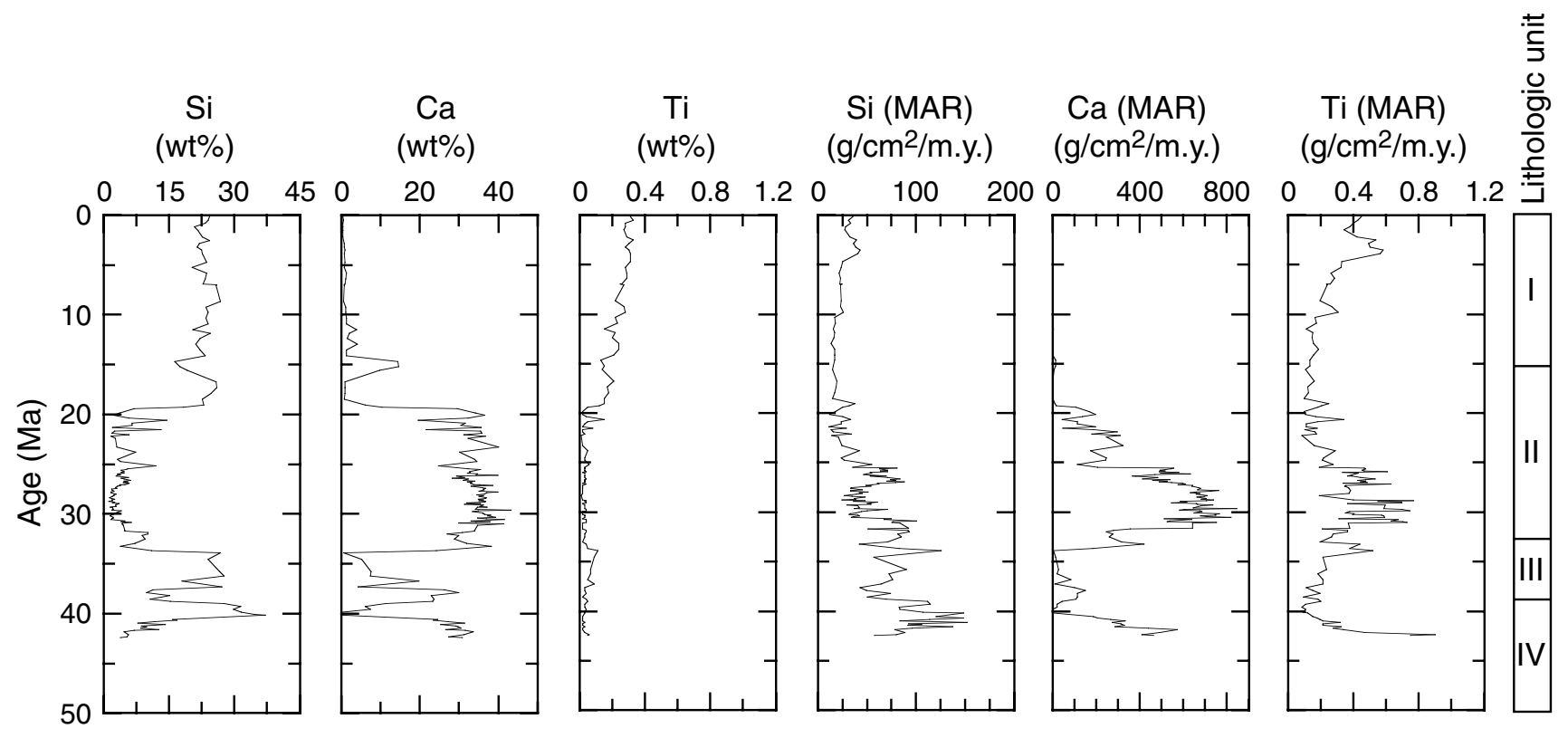


L.L. QUINTIN ET AL.

Chapter 7, Geochemical Analysis of Bulk Marine Sediment

Table T1. Analytical information from Leg 199.

\begin{tabular}{lcccccc}
\hline Element & $\begin{array}{c}\text { Wavelength } \\
(\mathrm{nm})\end{array}$ & $\begin{array}{c}\text { Detection } \\
\text { limit }(\mathrm{wt} \% \\
\text { or ppm) }\end{array}$ & $\begin{array}{c}\text { Within-run } \\
\text { drift } \\
(\%)^{\star *}\end{array}$ & $\begin{array}{c}\text { Within-run } \\
\text { precision } \\
(\%)^{\dagger \dagger}\end{array}$ & $\begin{array}{c}\text { Within-site } \\
\text { precision } \\
(\%)^{\ddagger \ddagger}\end{array}$ & $\begin{array}{c}\text { Long-term } \\
\text { precision } \\
(\%)^{\star \star \star}\end{array}$ \\
\hline $\mathrm{Si}$ & 251.611 & 0.1 & 1.2 & 1.7 & 0.9 & 3.3 \\
$\mathrm{Al}$ & 396.152 & 0.02 & 1.1 & 0.8 & 3.8 & 5.1 \\
$\mathrm{Ti}$ & 334.941 & 0.002 & 0.5 & 0.6 & 1.3 & 21.7 \\
$\mathrm{Fe}$ & 259.940 & 0.003 & 2.9 & 0.9 & 2.4 & 9.2 \\
$\mathrm{Mn}$ & 257.610 & 0.001 & 0.8 & 1.2 & 1.9 & 8.1 \\
$\mathrm{Ca}$ & 393.366 & 0.012 & 1.7 & 0.9 & 0.7 & 11.5 \\
$\mathrm{Mg}$ & 285.213 & 0.003 & 0.5 & 7.6 & 2.3 & 12.7 \\
$\mathrm{P}^{\star}$ & 178.229 & 0.07 & 30.8 & 68.5 & 67.8 & 32.9 \\
$\mathrm{P}^{\dagger}$ & 178.229 & 0.07 & 11.2 & - & 8.6 & - \\
$\mathrm{Ba}$ & 455.403 & 3.9 & 1.7 & 0.3 & 2.8 & 21.3 \\
$\mathrm{Sr}$ & 407.771 & 1.7 & 1.3 & 1.0 & 1.9 & 14.7 \\
\hline
\end{tabular}

Notes: * $=$ phosphorus analyses using the nitrogen generator, Site 1218. $\dagger=$ phosphorous analyses using the nitrogen storage tank, Site 1221. $\ddagger=$ detection limits calculated as three times the standard deviation of the blank: $\mathrm{Si}, \mathrm{Al}, \mathrm{Ti}, \mathrm{Fe}, \mathrm{Mn}$, $\mathrm{Ca}, \mathrm{Mg}$, and $\mathrm{P}$ listed in weight percent, and $\mathrm{Ba}$ and $\mathrm{Sr}$ listed in ppm. ** $=$ representative drift values within a single analytical run, Site $1218 . \dagger \dagger=$ representative precision for a single typical analytical run using JCh-1, Site 1218 . $\ddagger \neq=$ representative precision using drift solution, Site $1218 .{ }^{* * *}=$ based on precision values, Sites 1215-1221. 